Technical note

\title{
Proteomic profiling reveals sub proteomes of the human placenta
}

\author{
Wendy E. Heywood ${ }^{b}$, Rhian-Lauren Preece ${ }^{a}$, Jeremy Pryce ${ }^{a}$, Jenny Hallqvist ${ }^{b}$, \\ Robert Clayton ${ }^{\mathrm{b}}$, Alex Virasami ${ }^{\mathrm{a}}$, Kevin Mills ${ }^{\mathrm{b}}$, Neil J. Sebire ${ }^{\mathrm{a}, *}$ \\ ${ }^{a}$ Histopathology Department, Great Ormond Street Hospital for Children, Great Ormond Street, London, WC1N 3JH, UK \\ ${ }^{\mathrm{b}}$ Centre for Translational Omics, UCL Great Ormond Street Institute of Child Health, 30 Guilford Street, London, WC1N 1EH UK
}

\section{A R T I C L E I N F O}

\section{Article history:}

Received 14 August 2017

Received in revised form 25 September 2017

Accepted 28 September 2017

\section{Keywords:}

Proteomics

Placenta

Chorion

Decidua

Trophoblast

\begin{abstract}
A B S T R A C T
Proteomic characterisation of the placenta has largely been focused on effect of disease, anatomical features or specific cell types. We describe an unbiased proteomic mapping analysis to investigate how the placental proteome changes throughout the organ. A transverse slice of a human placenta was sectioned into $1 \times 1 \mathrm{~cm}$ samples. Sections were analysed using label free proteomics. Analysis revealed two distinct sub-proteomes that did not have anatomical significance. One had a muscular proteome and the other had distinct immunomodulation functions. Chorionic plate enriched proteins highlighted the fetal tissues high energy requirements whilst mechanisms of the decidua observed included modulation of cortisone levels.
\end{abstract}

(C) 2017 The Authors. Published by Elsevier Ltd. This is an open access article under the CC BY-NC-ND license (http://creativecommons.org/licenses/by-nc-nd/4.0/).

\section{Introduction}

There is growing interest regarding mechanisms by which placental function/dysfunction can affect short and long-term outcomes in fetal and postnatal life [1]. Variation in gene expression [2] and the transcriptome [3] of the human placenta have been described revealing its diverse molecular variation. However, to date little has been published in relation to detailed documentation of regional variation or anatomical mapping of the placental proteome. The human proteome atlas is able to describe the placental proteome through transcriptomic analysis and immunohistochemistry in regards to known placental cell types [4] but not in relation to regional or anatomical variation. Placental proteome studies have largely been conducted in respect to identifying changes in disease such as preeclampsia and fetal growth restriction [5,6]. Many studies have used limited samples from poorly defined sites within the placenta, such as a 'cotyledon'. However, the tissue proteome of an organ can differ markedly according to sub-anatomical regions and functional variation, which can markedly affect methodologies for how proteomic studies should be performed. This study has investigated whether there is variation across the placenta and whether sub-proteomes of the human term placenta represent functional differences in relation to sites of the

\footnotetext{
* Corresponding author.

E-mail address: Neil.Sebire@gosh.nhs.uk (N.J. Sebire).
}

placenta proteome map.

\section{Methods}

\subsection{Detailed methods are provided in supplementary information}

A placenta from a clinically uncomplicated, term normal vaginal delivery was used for this study, donated for research with appropriate consent and ethical approval as part of a larger study examining placental sampling. The placenta was sectioned as shown in Fig. 1B within $24 \mathrm{~h}$ of delivery having been initially refrigerated at $4^{\circ}$. Placental protein was run on a 1D PAGE [7,8]and analysed using label free proteomics as described previously [9].

\section{Results \& discussion}

Total analysis identified 1096 quantifiable proteins of $>95 \%$ confidence. The extreme end sections which form the peripheral margin of the placenta were removed from the analysis; as the proteomic integrity of these end sections was poor indicating this area undergoes significant degradation post-delivery. To identify if there was anatomical clustering of profiles the data were examined using principle components analysis, which shows an overall view of the variation of the placental proteome (Fig. 1A). The PCA resulted in a four component model $(\mathrm{R} 2 \mathrm{X}(\mathrm{cum})=0.581$ and $\mathrm{Q} 2(\mathrm{cum})=0.126)$. The first and second component best described 


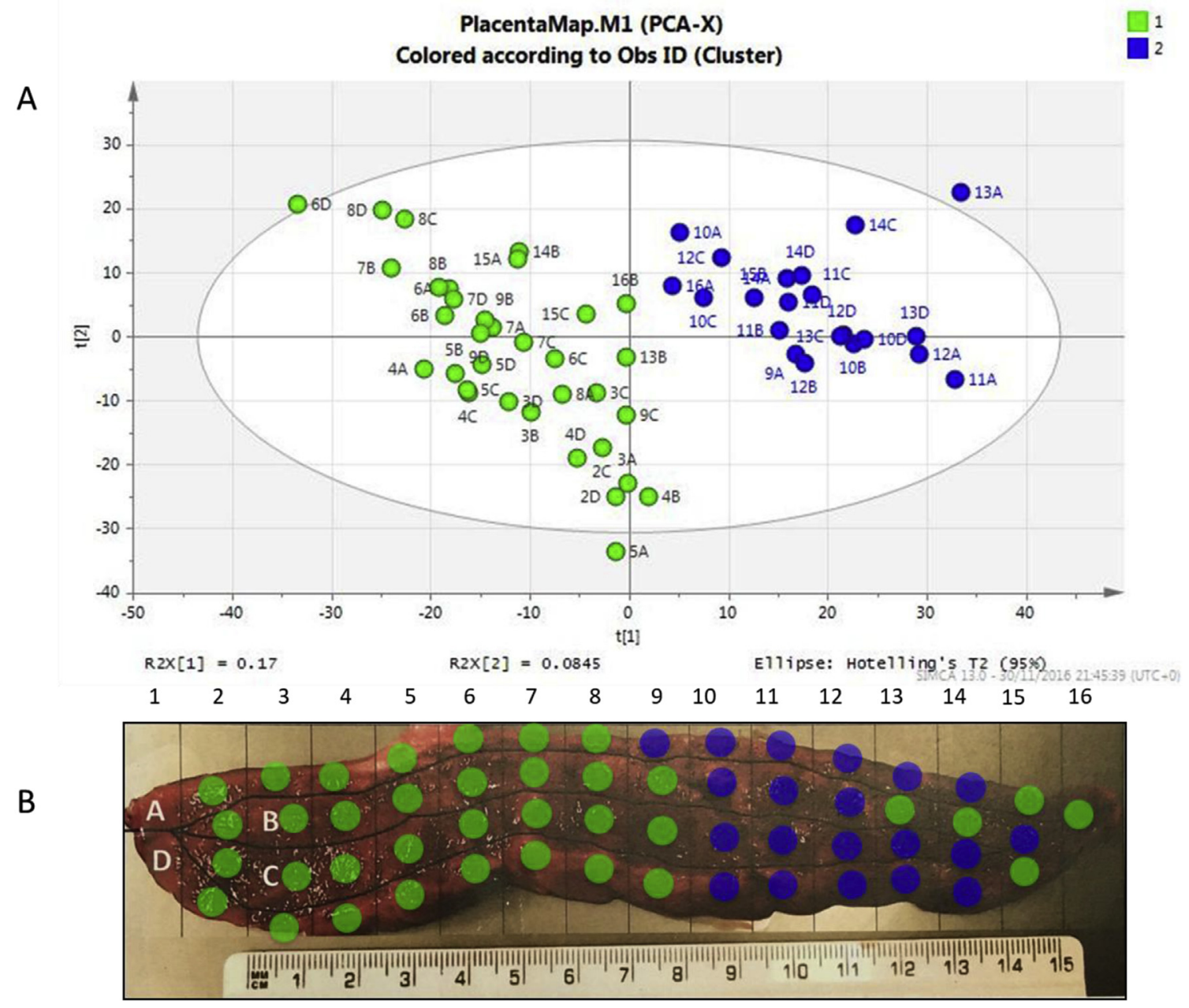

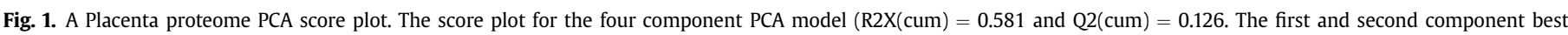

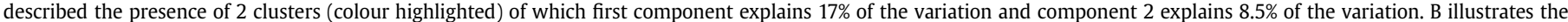
sectioning and depicted subproteome clusters identified in A annotated to the anatomical structure of the placenta.

the separation of two major sub-proteomes of the human placenta.

Gene Ontology analysis of clustered regions demonstrates that cluster 1 is enriched with proteins involved in suppression of the innate immune response, in particular antigen presentation. Pathway analysis (Supplementary data 4) also identifies antigen presentation as a significant pathway. Cluster 2 appears to have enriched proteins involved in exocytosis, muscle hypertrophy and the signalling cascade of SMAD protein import to the nucleus and protein localisation. Pathway analysis also confirms platelet degranulation (or exocytosis) and keratinisation as enriched. The list for cluster 2 also seems to show more extracellular matrix proteins. These analyses suggest presence of sub-proteomes with differing functions.

\subsection{Proteins that do not change throughout the placenta}

Proteins that show the least variation across the placenta as indicated by a coefficient of variation (CV) of $<30$ across all sections were proteins involved in integrity of the cytoskeleton and metabolism (supplementary data 2). As expected many other proteins with lower CV values are known housekeeping proteins. These proteins which have uniform expression throughout the placenta could potentially be used for standardising future analyses.

\subsection{Comparison by anatomical region; maternal vs fetal}

The overall placenta PCA analysis does not show marked clustering according to whether sections are from the maternal and fetal sides of the placenta, indicating that these proteomic differences are small, presumably since the majority of tissue represents parenchymal villi from all sites. To identify which proteins are altered between the maternal and fetal sides rows A and D were compared. Eleven proteins were altered in both clusters for which seven were enriched towards the maternal side and four at the fetal side (supplementary data 5). A selection of interesting differently expressed proteins are shown in a placenta 3D heat map in Fig. 2. These include; Corticosteroid 11-beta-dehydrogenase isozyme 2 which acts to inactivate cortisone which is an essential process in pregnancy. This enzyme is inhibited by liquorice (Glycyrrhiza uralensis) and may explain why ingestion of liquorice is not advised during pregnancy $[10,11]$. Elevated Tryptophan-tRNA ligase, cytoplasmic, may be related to involvement in angiogenesis and sheer stress response of the endothelium. Fetal side enriched proteins included the ECM component prolargin, creatine kinase B-type, required for tissues with high energy demands. cAMP-dependant protein kinase type II is involved in signal transduction in particular response to lipid and glucose metabolism and Apolipoprotein AI is a lipid transport protein. Interestingly, a recent proteomic 


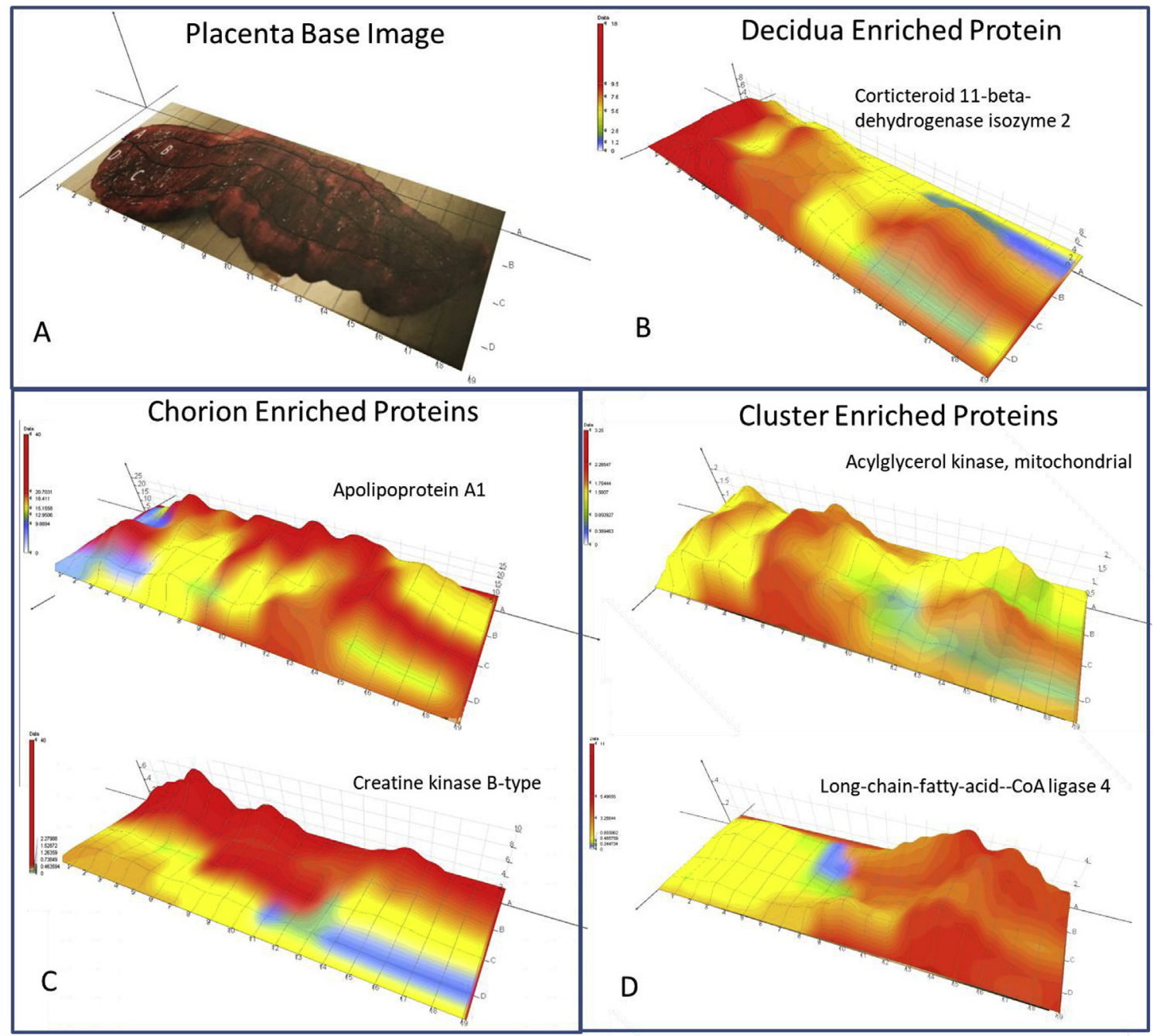

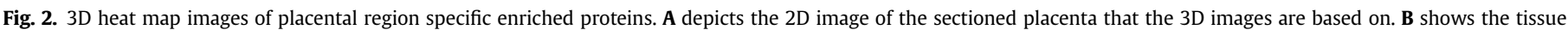

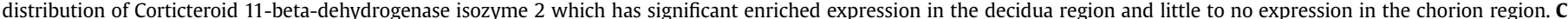

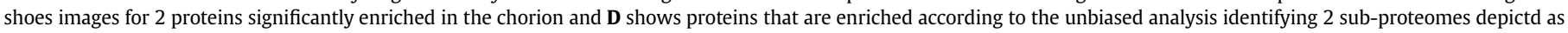
cluster 1 and 2 in Fig. 1.

study of preeclampsia reported that Apolipoprotein AI was elevated in maternal plasma of affected pregnancies [5] indicating this lipoprotein may play a key functional role during pregnancy. These enriched proteins suggest that energy metabolism is an important aspect at this site.

Significant fetal enriched proteins show enriched processes for actin arrangement and muscle filament sliding and contraction, keratinisation, embryo implantation and negative regulation of peptidase activity. The maternal aspect overall has a greater number of enriched proteins involved in processes such as antigen presentation, protein folding and modification energy metabolism. This report demonstrates proof of principle that the placenta proteome may be significantly affected by exact sampling site and that this may be of importance for future studies.

\section{Acknowledgements}

This work was supported by the National Institute for Health Research (NIHR) Biomedical Research Centre at Great Ormond Street Hospital for Children NHS Foundation Trust. The funders played no role in the performance or writing of the study and the views expressed are those of the authors and not the NIHR.

\section{Appendix A. Supplementary data}

Supplementary data related to this article can be found at https://doi.org/10.1016/j.placenta.2017.09.014.

\section{References}

[1] R.M. Lewis, J.K. Cleal, M.A. Hanson, Review: placenta, evolution and lifelong health, Placenta 33 (Suppl) (2012) S28-S32.

[2] R. Sood, J.L. Zehnder, M.L. Druzin, P.O. Brown, Gene expression patterns in human placenta, Proc. Natl. Acad. Sci. U. S. A. 103 (14) (2006) 5478-5483.

[3] D.A. Hughes, M. Kircher, Z. He, S. Guo, G.L. Fairbrother, C.S. Moreno, P. Khaitovich, M. Stoneking, Evaluating intra- and inter-individual variation in the human placental transcriptome, Genome Biol. 16 (2015) 54.

[4] M. Uhlen, L. Fagerberg, B.M. Hallstrom, C. Lindskog, P. Oksvold, A. Mardinoglu, A. Sivertsson, C. Kampf, E. Sjostedt, A. Asplund, I. Olsson, K. Edlund, E. Lundberg, S. Navani, C.A. Szigyarto, J. Odeberg, D. Djureinovic, J.O. Takanen, S. Hober, T. Alm, P.H. Edqvist, H. Berling, H. Tegel, J. Mulder, J. Rockberg, P. Nilsson, J.M. Schwenk, M. Hamsten, K. von Feilitzen, M. Forsberg, L. Persson, F. Johansson, M. Zwahlen, G. von Heijne, J. Nielsen, F. Ponten, Proteomics. Tissue-based map of the human proteome, Science 347 (6220) (2015) 1260419.

[5] S. Mary, M.J. Kulkarni, D. Malakar, S.R. Joshi, S.S. Mehendale, A.P. Giri, Placental proteomics provides insights into pathophysiology of pre-eclampsia and 
predicts possible markers in plasma, J. proteome Res. 16 (2) (2017) 1050-1060.

[6] P. Huuskonen, M.R. Amezaga, M. Bellingham, L.H. Jones, M. Storvik, M. Hakkinen, L. Keski-Nisula, S. Heinonen, P.J. O'Shaughnessy, P.A. Fowler, M. Pasanen, The human placental proteome is affected by maternal smoking, Reprod. Toxicol. 63 (2016) 22-31.

[7] P.B. Mills, K. Mills, A.W. Johnson, P.T. Clayton, B.G. Winchester, Analysis by matrix assisted laser desorption/ionisation-time of flight mass spectrometry of the post-translational modifications of alpha 1-antitrypsin isoforms separated by two-dimensional polyacrylamide gel electrophoresis, Proteomics 1 (6) (2001) 778-786.

[8] A. Shevchenko, M. Wilm, O. Vorm, M. Mann, Mass spectrometric sequencing of proteins silver-stained polyacrylamide gels, Anal. Chem. 68 (5) (1996)
$850-858$.

[9] U. Distler, J. Kuharev, P. Navarro, Y. Levin, H. Schild, S. Tenzer, Drift timespecific collision energies enable deep-coverage data-independent acquisition proteomics, Nat. methods 11 (2) (2014) 167-170.

[10] J.S. Choi, J.Y. Han, H.K. Ahn, H.M. Ryu, M.Y. Kim, J.H. Chung, A.A. Nava-Ocampo, G. Koren, Fetal and neonatal outcomes in women reporting ingestion of licorice (Glycyrrhiza uralensis) during pregnancy, Planta Med. 79 (2) (2013) 97-101.

[11] K. Raikkonen, J.R. Seckl, K. Heinonen, R. Pyhala, K. Feldt, A. Jones, A.K. Pesonen, D.I. Phillips, J. Lahti, A.L. Jarvenpaa, J.G. Eriksson, K.A. Matthews, T.E. Strandberg, E. Kajantie, Maternal prenatal licorice consumption alters hypothalamic-pituitary-adrenocortical axis function in children, Psychoneuroendocrinology 35 (10) (2010) 1587-1593. 